\title{
Association of MITF gene with hearing and pigmentation phenotype in Hedlund white American mink (Neovison vison)
}

\author{
MARIOS N. MARKAKIS ${ }^{1}$, VIBEKE E. SOEDRING ${ }^{2}$, VIBEKE DANTZER ${ }^{2}$, KNUD CHRISTENSEN $^{3}$ and \\ RAZVAN ANISTOROAEI ${ }^{3 *}$ \\ ${ }^{1}$ Department of Biology, Plant Growth and Development, University of Antwerp, 2020 Antwerp, Belgium \\ ${ }^{2}$ Section of Anatomy and Biochemistry and ${ }^{3}$ Section of Genetics, Bioinformatics and Systems Biology, Department of \\ Veterinary Clinical and Animal Sciences, University of Copenhagen, 1870 Frederiksberg C, Denmark
}

[Markakis M. N., Soedring V. E., Dantzer V., Christensen K. and Anistoroaei R. 2014 Association of MITF gene with hearing and pigmentation phenotype in Hedlund white American mink (Neovison vison). J. Genet. 93, 477-481]

Microphthalmia-associated transcription factor $(M I T F)$ is an important regulatory factor involved in the migration of melanocytes during embryonic development. Similar to other described species, in the American mink too, the MITF gene seems to be associated with lack of pigment and deafness, although no significant mutation has been identified in the gene. By histochemical methods, melanin granules were for the first time revealed in the stria vascularis in $w t$ mink but also in the vestibular membrane, a location never reported before. In the cochleas from deaf Hedlund mink ( $h h)$, no staining was observed.

The Hedlund white (Hedlund) phenotype in American mink (Neovison vison) (figure 1A) is similar to the Regal white (albino) type (Anistoroaei et al. 2008) but the eyes are dark, most often blue, and the animals are deaf. The skin of the newborn Hedlund kits is pale pink, but soon after birth is covered completely with white hair. The Hedlund phenotype in American mink appears to be controlled by a simple incompletely recessive locus and is associated with deafness in homozygotic status, similar to MITF variants in other species. The heterozygous $(H h)$ carriers have white markings on the belly, tail and paws (figure 1B).

Pigmentation defects resulting in either white or spotted phenotypes, sometimes associated with total or partial deafness have been described in cat, dog (Stritzel et al. 2009), cattle (Philipp et al. 2011), horse (Hauswirth et al. 2012) and humans (Tietz syndrome (TS) (http://omim.org/ entry/103500); Waardenburg syndrome type IIA (WSIIA) (http://omim.org/entry/193510); and 13 other OMIM entries at NCBI). The MITF gene was found to be associated

*For correspondence. E-mail: ran@sund.ku.dk. with these syndromes and/or mutated. MITF, except when expressed in the retina or in the neurons in the brain, is implicated in the migration of neural-crest-derived melanoblasts across the entire body surface. It transactivates the gene for tyrosinase (TYR), and is critically involved in melanocyte differentiation (Tachibana et al. 1996; Tachibana 2000) during foetal development (Breathnach 1988). The absence of melanocytes affects pigmentation in the skin, hair and hearing function in the cochlea. Common to nearly all MITF mutations are defects in neural-crest-derived melanocytes, manifested by a lack of pigment in the coat and inner ear. Many mutations also produce defects in retinal pigment epithelial (RPE) cells, which in turn results in abnormal eye development. This condition is however not observed in the American mink, where the eyes are dark (figure 1A) and blue iris colour is only an occasional finding. A histological study of melanin pigment in the stria vascularis from pigmented and white animals of five species showed variable amounts and localization of melanin pigments in stria vascularis in the pigmented animals, whereas no melanin was found in the albino type inner ear (Conlee et al. 1989). With electron microscopy (EM), Steel and Barkway (1989) studied the stria vascularis in mutant mice with dominant variable spotting, and suggested that melanocyte-like cells are vital for normal development of the stria vascularis. In a histochemical study using light and transmission EM, the total absence of the stria melanocytes and hearing acuity was found in double homozygous micropthalmic mice. The loss of hearing ability in the micropthalmic mice could be regarded as a dysfunction of the stria vascularis due to absence of melanocytes and thus impaired ion transport, inducing a low endolymphatic pressure (Motohashi et al. 1994). Disruption of MITF causes hearing impairment, heterochromia irides and leucodermia in WSIIA humans and deafness, micropthalmia

Keywords. American mink; Hedlund white; albino; deafness; MITF gene. 


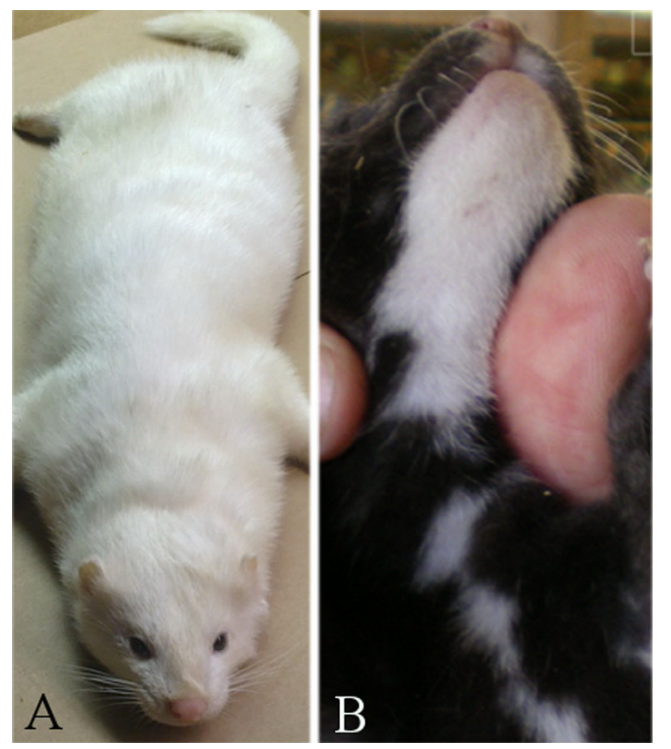

Figure 1. (A) A homozygous Hedlund white female mink, where the black eyes can be noticed. (B) A heterozygote puppy $(H h)$ with white irregular spots along the abdominal midline.

and white colour in mice. Many signal transduction pathways target the MITF gene or MITF protein, thus disruptions of these pathways may result in phenotypes similar to that caused by MITF disruption (Tachibana 2001). MITF is an important regulatory component in transcriptional network of many developmental pathways (reviewed by Hallsson et al. 2007).

Genomic sequencing of MITF in vertebrates has revealed a complex intron-exon structure with several widely spaced $5^{\prime}$ exons which are expressed from different promoters, giving rise to protein products with different $\mathrm{N}$-termini. This was demonstrated to be important for tissue-specific expression and to affect the transcriptional activation potential of the resulting proteins. In addition to alternative usage of $5^{\prime}$ exons, splice forms have been described that would lead to different MITF proteins through the alternative uses of exons 2 through 9 (reviewed by Hallsson et al. 2007). Our investigation took into consideration only the M-promoter, as we considered it to be the most relevant for the involvement in pigmentation mechanisms (as indicated by Karlsson et al. 2007).

The MITF gene in many studied species has over $3 \mathrm{~kb}$ of untranslated region (UTR) at the $3^{\prime}$ end, extending from the stop codon in exon 9 to the polyA sequence of the cDNA. Comparison of the $3^{\prime}$ UTR region between several species revealed a high conservation at a number of potential miRNA target sites suggesting a functional role in gene regulation (Hallsson et al. 2007). The role of miRNAs in MITF regulation is however largely unknown.

The many mutations at the mouse MITF locus have provided important insights into the structure-function relationship of this transcription factor. All mouse MITF mutations affect melanocytes to a varying degree, resulting in animals with coat colour phenotypes ranging from white spotting and coat colour dilution to a completely white coat due to lack of melanocytes (reviewed by Steingrimsson et al. 2004). Some of the mutations in mice also affect RPE cells, resulting in unpigmented or hypopigmented microphthalmic eyes but also osteoclasts, mast cells as well as natural killer cells, basophils, macrophages and B cells (reviewed by Steingrimsson et al. 2004), conditions which have not been documented in mink. In Fleckvieh cattle, a dominantly inherited syndrome associated with hypopigmentation, heterochromia irides, colobomatous eyes and bilateral hearing loss, a clear-cut missense mutation within MITF (p.R210I) has been identified to be responsible (Philipp et al. 2011). In Syrian hamster with a syndrome similar to Waardenburg type II, a point mutation in intron 6, 16-bp upstream exon 7, in a region highly conserved between species, induces the skipping of exon 7 with frameshifting of the transcript and its early termination (Graw et al. 2003). In these contexts, MITF was the most suitable candidate for congenital sensorineural deafness related to pigmentation status in Hedlund, as investigated in this study.

\section{Methods}

Based on the analysis of the assembled MITF gene genomic contig (JF288172), derived from the CHORI-231 American mink BAC library (Anistoroaei et al. 2011), the mink 1M variant of the MITF gene is predicted to be represented by nine exons and 4515 nucleotides. The coding region consists of $1260 \mathrm{bp}$, the $5^{\prime} \mathrm{UTR}$ region encompasses $134 \mathrm{bp}$ while the $3^{\prime}$ UTR consists of 3121 bp. From the JF288172 contig, two microsatellite markers, RAN98 and RAN99 (table 1 in electronic supplementary material at http://www.ias.ac.in/ jgenet/) were indentified. These markers were used for genotyping within one half-sib family of 25 individuals from a farm in Western Denmark, showing segregation for the white/deafness phenotype (figure 1 in electronic supplementary material).

\section{DNA and genotyping protocol}

DNA was extracted from the nails using the Chelex method (Walsh et al. 1991). Genotyping was performed with fluorescently labelled forward primers on an ABI 3130 sequencer and analysed using the Genscan Analysis (ver. 3.1.2) software (Applied Biosystems, Foster City, USA). Cri-map analysis was applied to test for linkage between the genotypes of the two microsatellite markers and the white/deaf phenotype.

\section{Histological analysis preparations}

The ventro-caudal part of the cranium with pars petrosa of the temporal bone with left and right sides were excised and two small punctures were made in the bulla ossea 
which before immersion was fixed in $4 \%$ buffered formalin, with a total fixation time of $48 \mathrm{~h}$. The blocks were then incubated in 6\% EDTA for decalcification for three months and subsequently trimmed, orientated and embedded in paraffin by routine methods. To visualize sections of the cochlea they were stained with HE. Serial sections were subsequently produced and selected section from these serial sections were stained with Masson-Fontana and Schmorl (Stevens 1982) to demonstrate melanin granules in the stria vascularis of the cochlear duct, where the endolymph is synthetized. No counterstain was done to facilitate visualization. A positive control originated from coloured hairy skin of the external ear from a wt mink. Micrographs were taken by means of a Leica DMR (Leica, Wetzlar, Germany).

\section{Results and discussion}

Results from the genotyping of $R A N 98$ and $R A N 99$, after the Cri-map analysis revealed informative polymorphisms for both these markers, yielding a LOD score value of 6 and 5, respectively, and perfect cosegregation with the white/deaf phenotype. Thus, as in the case of other mammalian species where MITF mutations are causing phenotypes with loss of pigmentation and hearing, an involvement of MITF appears also in Hedlund mink.

For sequence analysis of the MITF gene, we initially designed primers on the flanking intronic regions of all nine exons of the mink (JF288176; Anistoroaei et al. 2011). After PCR amplification, the DNA products were sequenced and sequence comparison was performed between a single Hedlund individual, a $w t$ mink and the reference sequence (JF288176) in order to detect relevant polymorphisms. The search and comparison was subsequently expanded to the long 3'UTR of the MITF gene. The processed sequences were compared using the BLAST bl2seq at NCBI. Gradually, as no relevant mutation was revealed, we expanded our investigation upstream along the coding region, encompassing more than 5000-bp upstream the exon $1 \mathrm{M}$ (see figure $2 \mathrm{~b}$ in electronic supplementary material). Investigation of this region was suggested by a finding described by Karlsson et al. (2007) in which following a genomewide association study in dog, two mutations located about 3000-bp upstream of the M promoter of MITF and a sequence length polymorphism upstream of the TATA box were found to be associated with extreme white coat colour and flash phenotypes in dogs.

For illustrative purposes, we indicate the primers in two figures backed up by corresponding tables (figures $2 \& 3$; tables $1 \& 2$ in electronic supplementary material). Intronic amplicons of MITF were not sequenced, but used to comparatively check for large size differences between Hedlund and $w t$, by running them on a high resolution agarose gel (MetaPhor Agarose, Cambrex, Rockland, USA).

Our investigations in the coding region of the MITF in mink did not reveal any mutations/polymorphisms between Hedlund versus $w t$ and the reference contig (JF288172)
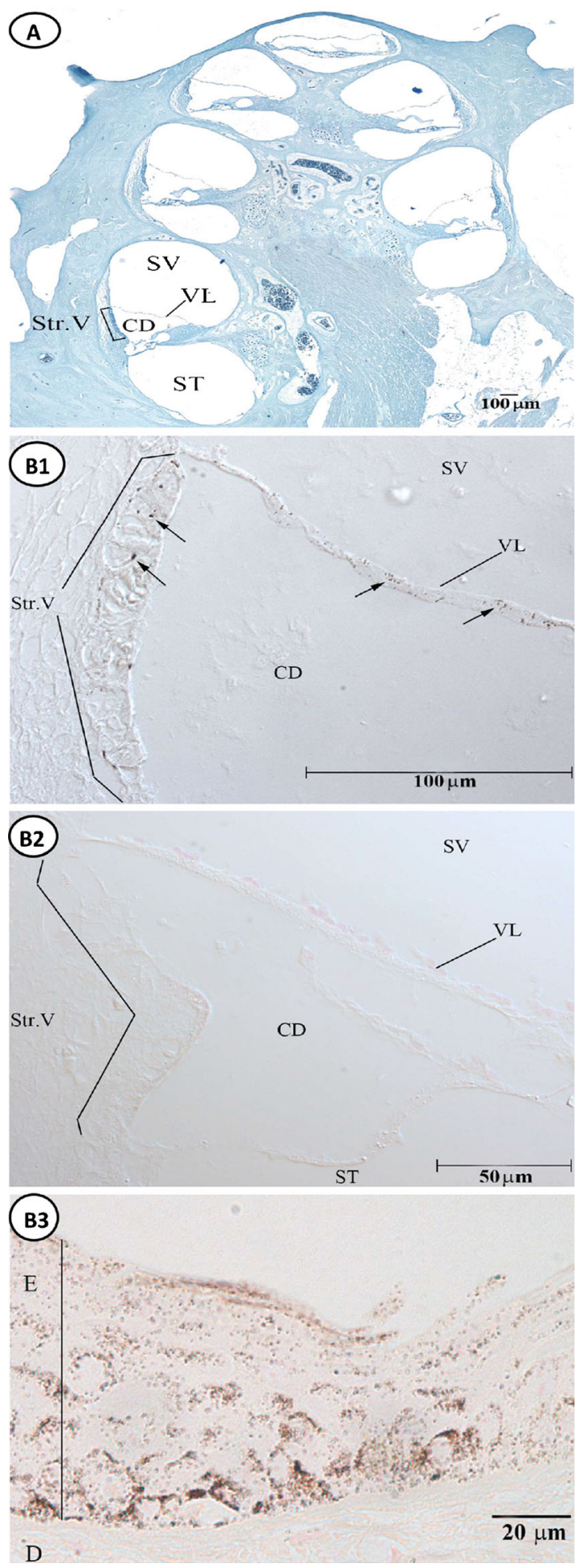

Figure 2. (A) An overview of a control cochlea showing the cochlear duct (CD) with endolymph and the scala vestibuli (SV) and scala tympani (ST) with perilymph. The stria vascularis (Str.V) is located in the outer wall of the cochlear duct. The vestibular ligament (VL) separates the cochlear duct from the SV. Schmorl staining. (B) Detail from cochlea in: (1) black control mink $(H H)$; (2) deaf Hedlund white $(h h)$; (3) positive control of hairy skin with melanocytes and stained melanin granules. Masson-Fontana staining. DC, cochlear duct; E, stratified epithelium; D, dermis; arrows; stained melanin granules. 
respectively. Further, parts of intronic sequences flanking the exons (at least $50 \mathrm{bp}$, see figure 2; table 1 in electronic supplementary material) were also evaluated for polymorphisms. In all the cases, including these regions earlier described as ultraconserved between species (Graw et al. 2003), no differences were observed.

Additionally, the fact that the $3^{\prime}$ UTR of the MITF gene has at least six binding sites for miRNAs which seem to be very well-conserved among species (Hallsson et al. 2007), made us follow and sequence the entire $3^{\prime} \mathrm{UTR}$ of MITF in mink. Interestingly, the $w t$, as derived both from the MITF contig assembly (JF288172) and from two $w t$-sequenced individuals, contains a variation (c. ${ }^{*} 2970 \mathrm{C}>\mathrm{T}$ ) at an ultraconserved miR site of the $3^{\prime}$ UTR (figure 4 in electronic supplementary material). The macroanalysis of the intron large variations did not reveal any differences at this level. All these results suggest that the mutation(s) could be more complex requiring other types of analysis (e.g. qPCR, expression studies) for this regulatory gene in mink.

For histological analysis purposes, following euthanasia, heads from two wt mink with normal auditive capability/hearing acuity (controls) two Hedlund $(h h)$ with profound hearing impairment were prepared. A detailed protocol is presented in methods.

Routine staining with HE showed melanin granules in skin but not in the stria vascularis of cochlear duct. By the two histochemical methods, melanin granules were for the first time revealed in the stria vascularis in $w t$ showing dark blue granules of different sizes by Schmorl staining (figure 2A) and black granules after Masson Fontana staining (figure 2B). In the cochleas from deaf Hedlund mink (hh) no staining was observed (figure 2B). In the positive control of skin, a strong precipitation was observed (figure $2 \mathrm{~B}$ ). We also found melanin granules in the vestibular membrane. This location seems not to have been reported before. However, Wright and Lee (1989) described chinchilla (Chinchilla chinchilla) with typical melanocytes in the stria vascularis as well as in the spiral ligament. A possible function of melanocytelike cells in this location has not been investigated. In mice, the close relation between hearing impairment, missing melanocytes and missing endocochlear potential (EP) has recently been reviewed (Hibino et al. 2010). EP is dependent on an active ion-transport apparatus in melanocyte-like cells and defects in this system cause hearing impairment (Steel and Barkway 1989; Hibino et al. 2010). Stria vascularis from mutant mice with impaired hearing was studied by transmission electron microscopy. Their results indicate a reduction in the height of the epithelium of stria vascularis due to the absence of melanocyte-like cells in the mutant mice (Steel and Barkway 1989). In our histochemical study for light microscopy it was however not possible to make this accurate comparison between stria vascularis from $w t$ and Hedlund mink. Together with results from other studies, our result strongly indicate that neural crest cell migration has been impaired during embryonic life in the Hedlund, as there is no pigment in the stria vascularis of deaf Hedlund and as they usually have pigmented eyes. However, in Hedlund, the migration of melanocytes in the embryonic stage is defective, unlike the Albino phenotype where the melanocytes are present but the formation of pigment is absent due to a tyrosinase deficiency (Anistoroaei et al. 2008).

In conclusion, this study indicates strong association between MITF gene and the Hedlund phenotype in mink. In addition, this is the first histochemical analysis of the deaf Hedlund mink revealing the lack of melanocytes in the stria vascularis of the inner ear.

\section{Acknowledgements}

This work was supported by Razvan Anistoroaei's postdoctoral grant from the Danish Research Council (\#27820) and funding from the Danish Fur Breeders Association. We are thankful to C.A. Halberg, M. Jakobsen and A. Strandsby for the technical assistance.

\section{References}

Anistoroaei R., Fredholm M., Christensen K. and Leeb T. 2008 Albinism in the American mink (Neovison vison) is associated with a tyrosinase nonsense mutation. Anim. Genet. 39, 645-648.

Anistoroaei R., ten Hallers B., Nefedov M., Christensen K. and de Jong P. 2011 Construction of an American mink bacterial artificial chromosome (BAC) library and sequencing candidate genes important for the fur industry. BMC Genomics 12, 35.

Breathnach A. S. 1988 Extra-cutaneous melanin. Pigm. Cell Res. 1(4), 234-237.

Conlee J. W., Parks Y. N., Schwartz I. R. and Creel D. J. 1989 Comparative anatomy of melanin pigment in the stria vascularis. Evidence for distinction between melanocytes and intermediate cells in the cat. Acta Otolaryngol. 107, 48-58.

Graw J., Pretsch W. and Löster J. 2003 Mutation in intron 6 of the hamster Mitf gene leads to skipping of the subsequent exon and creates a novel animal model for the human Waardenburg syndrome type II. Genetics 164, 1035-1041.

Hallsson J. H, Haflidadóttir B. S., Schepsky A., Arnheiter H. and Steingrímsson E. 2007 Evolutionary sequence comparison of the Mitf gene reveals novel conserved domains. Pigment Cell Res. 20, 185-200.

Hauswirth R., Haase B., Blatter M., Brook S. A., Burger D., Drögemüller D. et al. 2012 Mutations in MITF and PAX3 cause 'splashed white' and other white spotting phenotypes in horses. PLoS Genet. 8, e1002653.

Hibino H., Nin F., Tsuzuki C. and Kurachi Y. 2010 How is the highly positive endocochlear potential formed? The specific architecture of the stria vascularis and the role of the ion-transport apparatus. Eur. J. Physiol. 459, 521-533.

Karlsson E. K., Baranowska I., Wade C. M., Salmon Hillberg N. H., Zody M. C., Anderson N. et al. 2007 Efficient mapping of Mendelian traits in dogs through genome-wide association. Nat. Genet. 39, 1321-1328.

Motohashi H., Hozawa K., Oshima T., Takeuchi T. and Takasaka T. 1994 Dysgenesis of melanocytes and cochlear dysfunction in mutant micropthalmia (mi) mice. Hear. Res. 80, 10-20.

Philipp U., Lupp B., Mömke S., Stein V., Tipold A., Eule J. C., Rehage J. and Distl O. 2011 A MITF mutation associated with a dominant white phenotype and bilateral deafness in German Fleckvieh cattle. PLoS One 6, e28857.

Steel P. K. and Barkway C. 1989 Another role for melanocytes: their importance for normal stria vascularis development in the mammalian inner ear. Development 107, 453-463. 
Steingrimsson E., Copeland N. G. and Jenkins N. A. 2004 Melanocytes and the microphthalmia transcription factor network. Ann. Rev. Genet. 38, 365-411.

Stevens A. 1982 Pigments and minerals. In theory and practice of histological techniques (ed. J. D. Bancroft and A. Stevens), pp. 242-266. Churchill Livingston, Edinburgh, Scotland.

Stritzel S., Wöhlke A. and Distl O. 2009 A role of the microphthalmia-associated transcription factor in congenital sensorineural deafness and eye pigmentation in Dalmatian dogs. J. Anim. Breed. Genet. 126 1, 59-62.

Tachibana M. 2000 MIFT: a stream flowing for pigmented cells. A review. Pigm. Cell Res. 13, 230-240.
Tachibana M. 2001 Cochlear melanocytes and MITF signaling. $J$. Investig. Dermatol. Symp. Proc. 6, 95-98.

Tachibana M., Takeda K., Nobukuni Y., Urabe K., Long J. E. et al. 1996 Ectopic expression of MITF, a gene for Waardenburg syndrome type 2, converts fibroblasts to cells with melanocytes characteristics. Nat. Genet. 14, 50-54.

Walsh P. S., Metzger D. A. and Higuchi R. 1991 Chelex 100 as a medium for simple extraction of DNA for PCR-based typing from forensic material. Biotechniques 10, 506-513.

Wright C. G. and Lee D. H. 1989 Pigmented cells of the stria vascularis and spiral ligament of the chinchilla. Acta Otolaryngol. 108, 190-200.

Received 17 October 2013, in revised form 11 December 2013; accepted 12 December 2013

Published online: 24 July 2014 